\title{
Ongoing Response in BRAF V600E-Mutant Melanoma After Cessation of Intermittent Vemurafenib Therapy: A Case Report
}

\author{
Andrew J. Dooley ${ }^{2} \cdot$ Avinash Gupta $^{1} \cdot$ Mark R. Middleton $^{1}$
}

Published online: 9 February 2016

(C) Springer International Publishing Switzerland 2016

\begin{abstract}
The selective BRAF inhibitors vemurafenib and dabrafenib yield high response rates and improved overall survival in patients with BRAF V600E-mutant metastatic melanoma. Treatment traditionally continues until disease progression or the development of unacceptable toxicity. Acquired drug resistance and toxicity are key challenges with the use of these drugs. Resistance to vemurafenib usually develops within 68 months. Management of drug toxicity typically involves stopping vemurafenib until resolution, before restarting at a lower dose, or permanently ceasing vemurafenib therapy. We have recently considered whether intermittent dosing could be used as an alternative to dose reduction/termination in the management of vemurafenib toxicity. One patient treated with intermittent vemurafenib was an 89-year-old woman with metastatic melanoma, who initially showed a good response to continuous dosing. Recurrent toxicity meant that the continuous vemurafenib dosage was repeatedly ceased before restarting at a lower dose. Ten months after vemurafenib was first begun, an intermittent dosing regimen was introduced in an attempt to control toxicity. This continued for 2 months, before cessation due to continued unacceptable toxicity. A further 24 months later, the patient remains fit and well in complete clinical remission, with no recurrence of her previous melanoma and no new primary malignancies. To the best of our knowledge, a continued response after the cessation of selective BRAF inhibitors has
\end{abstract}

Mark R. Middleton

mark.middleton@oncology.ox.ac.uk

1 Department of Oncology, NIHR Biomedical Research Centre, Oxford Cancer and Haematology Centre, Churchill Hospital, Old Road, Headington, Oxford OX3 7LE, UK

2 University of Oxford, John Radcliffe Hospital, Oxford OX3 9DU, UK never before been described in melanoma. Induction of an immune response and/or epigenetic changes could explain continued disease response after cessation of vemurafenib therapy. Care should be taken when extrapolating the findings from the continued response after vemurafenib cessation to other tumour types. We recommend the collection and analysis of data to investigate the clinical responses seen after cessation of vemurafenib due to intolerable toxicities, which could help further explain vemurafenib's mechanism of action.

\section{Key Points}

The selective BRAF inhibitors, vemurafenib and dabrafenib, yield high response rates and improved overall survival in patients with BRAF V600E-mutant metastatic melanoma.

We have previously described the use of intermittent vemurafenib as an alternative to dose reduction/termination in the management of vemurafenib toxicity.

One patient previously described has shown a continued response after ceasing intermittent vemurafenib. A continued response after the cessation of selective BRAF inhibitors has never been previously described in melanoma.

\section{Background}

The v-raf murine sarcoma viral oncogene homolog B1 (BRAF) gene is mutated in 40-60\% of melanomas, the most common being the V600E mutation, which leads to overactivation of the mitogen-activated protein kinase (MAPK) pathway $[1,2]$. The selective BRAF inhibitors vemurafenib and dabrafenib yield high response rates and improved overall 
survival in patients with BRAF V600E-mutant metastatic melanoma [3, 4]. However, acquired drug resistance and drug toxicity are key challenges with the use of these drugs. Resistance to vemurafenib usually develops within 6-8 months [5]. In the BRAF Inhibitor in Melanoma-3 (BRIM-3) trial, a phase 3 randomised open-label study comparing vemurafenib with dacarbazine in BRAF V600E- and BRAF V600K-mutant melanoma, $38 \%$ of patients receiving vemurafenib required dose modifications because of toxicity [3]. Management of toxicity typically involves stopping vemurafenib until resolution, before restarting at a lower dose, or permanently ceasing vemurafenib therapy. In an extended follow-up of BRIM-3, treatment was discontinued because of adverse events in 24 patients receiving vemurafenib and six patients receiving dacarbazine. No data were presented on the outcome of these patients after cessation of therapy due to unacceptable toxicities [6]. Although disease progression has traditionally been seen as the point at which vemurafenib therapy should cease, recent research has suggested that treatment beyond progression might be beneficial [7]. Acquired resistance to targeted therapies is also a challenge in the treatment of non-small cell lung cancer (NSCLC). Epidermal growth factor receptor (EGFR) inhibitors such as gefitinib, erlotinib and axitinib are standard first-line treatments for patients with NSCLC with activating EGFR mutations [8]. Unfortunately, most patients eventually acquire resistance, and management options after progression on EGFR TKIs have yet to be defined $[9,10]$. The recent IMPRESS trial investigated whether targeted therapies should be continued after progression. The study concluded that continuation of gefitinib after radiological disease progression on first-line gefitinib did not prolong progressionfree survival in patients who received platinum-based doublet chemotherapy as a subsequent line of treatment [8].

We recently presented a case series in which patients with intolerable toxicities on a continuous dosing regimen of vemurafenib were treated instead with an intermittent regimen. Our experience showed that intermittent dosing could successfully manage vemurafenib toxicities where continuous dosing at a reduced dose did not, while maintaining or achieving melanoma shrinkage. We therefore recommended that intermittent dosing should be considered as an alternative to dose reduction/ termination in the management of vemurafenib toxicity [11]. While we could present no evidence that intermittent therapy is more effective than continuous therapy, or that it can prevent the appearance of drug-resistant disease, this is due to be investigated in clinical trials currently in set-up. In animal models, however, there is evidence which suggests that intermittent dosing of vemurafenib can forestall the emergence of resistance [12]. This study of an animal model of melanoma did not investigate whether intermittent dosing continued to exert an effect after cessation.

\section{The Case}

We previously reported the case of an 88-year-old woman diagnosed with metastatic melanoma who showed a good response to vemurafenib despite several dose reductions due to toxicity [11].

She originally had a superficial spreading melanoma removed from her left leg in 2006 when she was 80 years old. The primary melanoma had a Breslow thickness of $1.5 \mathrm{~mm}$ and was non-ulcerated, with a mitotic index of $0.1-0.5$ per $\mathrm{mm}^{2}$. No adjuvant therapy was given afterwards. A further melanoma was excised from the left leg in 2007 (again a superficial spreading melanoma, non-ulcerated, Breslow thickness $2.5 \mathrm{~mm}$, mitotic count 3 per $\mathrm{mm}^{2}$ ). The patient continued on surveillance follow-up. She remained well until 2011, when she developed multiple rapidly growing and painful subcutaneous lesions along her left leg, confirmed as melanoma on biopsy. A CT scan in November 2011 showed two 11-mm nodules in the right lung, suggesting small-volume metastatic disease. Her melanoma was confirmed as containing the BRAF V600E mutation, and she was commenced on vemurafenib at the standard starting dose of $960 \mathrm{mg}$ twice daily (BD) in January 2012, as part of an expanded access programme. A further CT scan just before starting treatment showed disease progression, with multiple small-volume pulmonary nodules and multiple enlarged left inguinal lymph nodes. Clinically, the disease in her left leg had also worsened. Pre-treatment LDH was elevated at $262 \mathrm{IU} / \mathrm{L}$ (normal range: 110-255).

The patient showed a rapid clinical response to therapy, with a reduction in the leg lesions within 4 weeks of starting treatment. However, she also experienced various toxicities, particularly joint pain and nausea. After 8 weeks of therapy, a CT scan showed the majority of pulmonary nodules and inguinal lymph nodes had either significantly shrunk or completely resolved. One nodule in the left lower lobe, however, had enlarged. A further CT scan after 16 weeks of therapy showed the left lower lobe pulmonary nodule remained unaltered with no new metastases. Clinically, the lesions in her left leg continued to shrink. However, by week 24 of therapy, she had lost $14 \mathrm{~kg}$ and developed grade 2 nausea and diarrhoea. Vemurafenib was ceased at this point. These toxicities quickly resolved, so therapy was restarted a week later at a lower dose of $720 \mathrm{mg}$ BD. However, the patient again developed toxicity within less than 2 weeks. Treatment was again ceased for 1 week, before restarting at $480 \mathrm{mg}$ BD. Treatment continued at this lower dose for 8 weeks, before a further 1week cessation due to toxicities. This pattern continued, with toxicity settling whilst off treatment but quickly re-emerging on restarting therapy. So, in November 2012, 10 months after initially starting vemurafenib, with CT and clinical evidence of ongoing response, a decision was made to recommence vemurafenib on an intermittent regimen of $480 \mathrm{mg} \mathrm{BD}, 1$ week on, 1 week off. As this could not be done within the 
expanded access programme, we applied for and received approval for this to be funded through the Cancer Drugs Fund. The lesions on the left leg continued to shrink on intermittent dosing, and 1 month later the leg was clear of all visible deposits. However, intermittent dosing was ceased in January 2013 because of grade 2 arthralgia in the patient's hands. A CT scan performed 4 weeks after cessation of vemurafenib showed an ongoing response, with no evidence of any metastatic disease. The marker left inguinal lymph node remained unchanged in size. We planned to restart vemurafenib as and when the leg lesions recurred, but so far this has not occurred. As of February 2015, the patient is alive and clinically free of disease. She has not needed any other treatment for her metastatic melanoma. A summary of her clinical history is presented in Table 1.

\section{Discussion}

To the best of our knowledge, a continued response after ceasing vemurafenib has not been previously described in metastatic melanoma. The melanoma showed a rapid response soon after starting vemurafenib on a continuous dosing regimen. This response continued on intermittent dosing, with the leg lesions continuing to shrink until they completely disappeared. We feared the disease would recur soon after vemurafenib was ceased. However, the complete response has continued for over 2 years after cessation of therapy. It is difficult to understand what role, if any, intermittent therapy played in this continued response, especially since such a rapid, although not universal, response to continuous dosing was seen. Although the patient is in complete clinical remission, this does not mean the melanoma has been completely eliminated. It is possible there are small areas of residual disease left in this patient, and recurrence could yet occur many years later. Since late recurrence of melanoma is not uncommon, no melanoma patient, including the one detailed in this report, can ever be considered cured [13].

\subsection{Continued Response in Non-Melanoma BRAF Mutated Cancer}

A continued response after ceasing vemurafenib therapy has been described in a patient with BRAF V600E-mutant hairy cell leukaemia (HCL) [14]. Briefly, this patient was treated with multiple lines of conventional therapy, but none proved effective. The presence of a V600E mutation was demonstrated, and so the patient was started on vemurafenib. The patient showed a rapid response to treatment within days, with a reduction in splenomegaly and rise in platelet, haemoglobin, and leukocyte counts. By day 35, flow cytometry showed no evidence of HCL cells in peripheral blood. The patient was treated with vemurafenib for a total of 56 days; the decision to cease therapy was made at this point because of the excellent response to treatment and to avoid cutaneous toxicities.
Despite discontinuation of vemurafenib, the patient remained in remission, with no evidence of minimal residual disease 6 months after stopping treatment. Analysis of trephine biopsies obtained before and during treatment demonstrated abolition of ERK phosphorylation in HCL cells within 6 days of starting vemurafenib, resulting in apoptosis of the leukemic cells [14]. These findings support the conclusions drawn by in vitro work performed by Tiacci et al., in which BRAF-mutated HCL cells were shown to be highly sensitive to BRAF inhibition [15].

In the BRAF-mutant HCL patient who showed a continued response after ceasing vemurafenib, the drug appeared to mediate its effect by inhibiting the MAPK pathway. This is the same pathway targeted by vemurafenib in BRAF-mutant melanoma [16]. However, the continued clinical responses seen in these two cases are not necessarily the same phenomena. In the HCL case, the rapid elimination of all detectable HCL cells suggests that vemurafenib is acting on a homogenous clonal population of BRAF-mutant HCL cells. In our patient with melanoma, the response in most lesions, but the increase and subsequent stabilisation in one pulmonary lesion, suggests activity against a more heterogenous tumour population.

Although it is likely that MAPK pathway inhibition is necessary for clinical effectiveness of vemurafenib in both melanoma and HCL, different biochemical pathways may be involved. The genetic basis of HCL is not fully understood, but pathways involving FLT3L and IL3 appear to play an important role in protecting HCL cells from apoptosis [17]. In melanoma, different pathways are involved, such as the CDKN2A/CDK4 system and pathways involving the extracellular matrix not found in HCL $[18,19]$. In the HCL case described above, it appears that BRAF inhibition interrupted signalling through MEK and ERK, which led to apoptosis in all of the clonal HCL cells, resulting (in this one case) in the effective elimination of the entire tumour population [14]. This suggests that all the BRAF-mutant HCL cells were dependent on aberrant BRAF signalling for survival. In BRAF-mutant melanoma, targeting the MAPK pathway alone is not sufficient to induce apoptosis in the entire tumour population. Much work on resistance to BRAF inhibition in melanoma has been done, and activation of the parallel PI3k/AKT pathway appears to play an important role [5, 20]. Although current understanding of $\mathrm{HCL}$ genetics is incomplete, the BRAF V600E mutation is found in the vast majority of HCL cases, compared with the 40-60\% of melanomas that contain a range of BRAF mutations $[1,21,22]$. Melanoma is the most mutated tumour in oncology, suggesting the presence of numerous alternate pathways that could play a role in resistance development [23].

There is now good evidence that melanoma metastases can be genetically heterogeneous, with genetic differences found both between and within different melanoma metastases in a single patient [24]. It is likely that different metastases will show different characteristics, both in terms of their natural history and in their response to vemurafenib. 


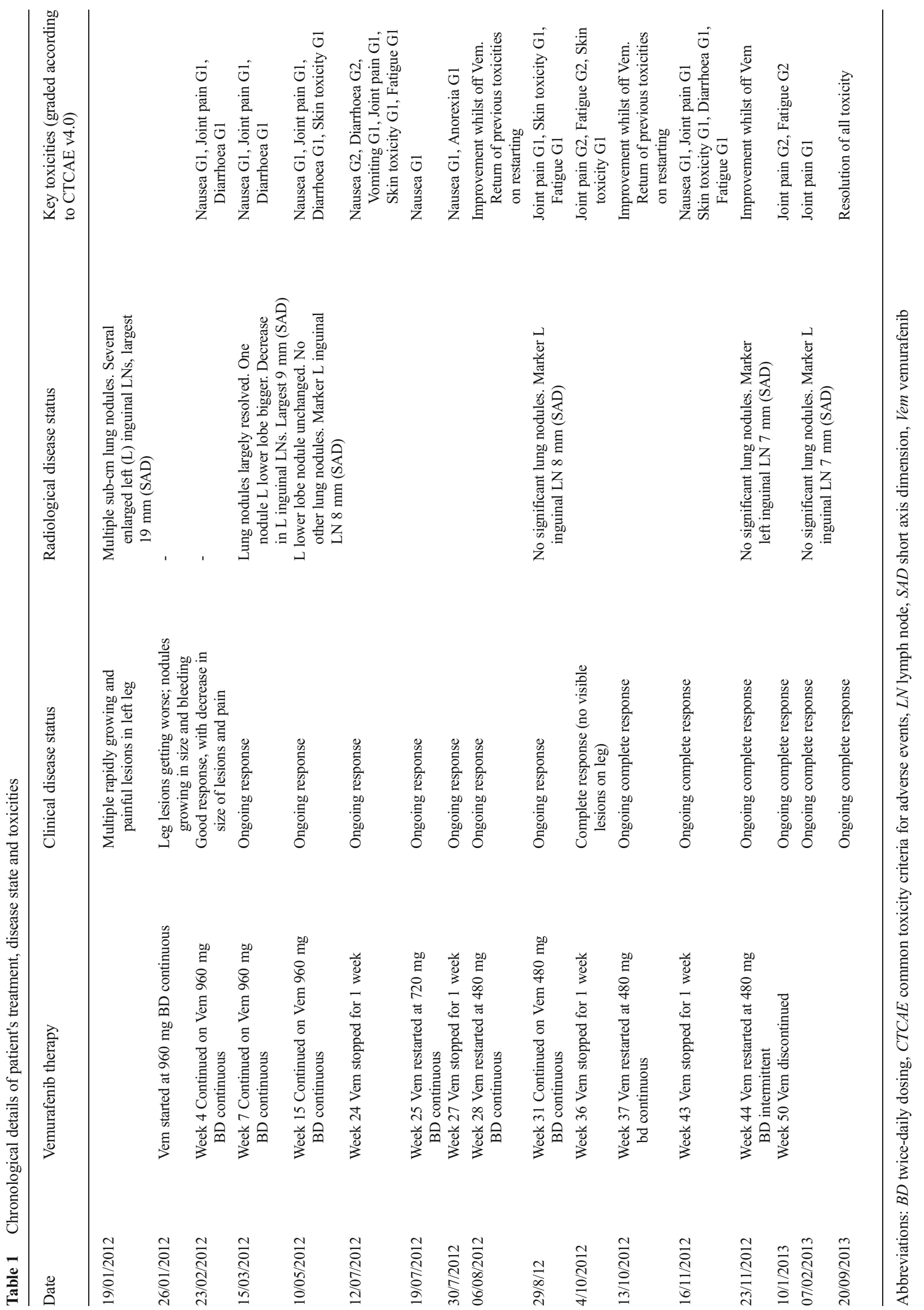


In this case report, the majority of melanoma metastases were very sensitive to vemurafenib, suggesting that the drug acted on these lesions directly through inhibition of ERK phosphorylation and subsequent apoptosis of cancer cells, as with the HCL case. However, the fact that one pulmonary lesion initially grew suggests it had less dependence on the MAPK pathway. The subsequent growth arrest with continued vemurafenib treatment suggests that the drug eventually worked via a different mechanism (possibly an immune-mediated response).

\subsection{Possible Explanations for the Continued Response}

There are several possible explanations as to why the disease did not progress after cessation of treatment in the case of our patient. Firstly, the natural history of the disease needs to be understood; it could be that this patient had an indolent form of melanoma. Our patient is female; there is a much more favourable long-term survival for women after development of distant metastases [25]. However, we feel that this is an unlikely explanation for this patient, given that prior to starting vemurafenib, the melanoma was growing rapidly, especially the leg lesions.

Other potential mechanisms by which vemurafenib could produce a lasting response, even after cessation of treatment, could include the induction of an immune response and/or epigenetic mechanisms.

\subsection{Epigenetic Modification}

It is increasingly understood that epigenetics plays a role in the pathogenesis of cancer, and several epigenetic modifiers are in development, where the drug targets enzymes involved in epigenetic control of gene expression. These targets include DNA methyltransferases and acetyltransferases. Epigenetic modifiers such as azacitidine (a DNA methyltransferase) and vorinostat (a histone deacetylase) are now approved cancer therapies [26]. Epigenetic modification of gene expression can result in the drug continuing to exert its effect long after its administration has been ceased [27]. It is plausible that BRAF inhibition could induce epigenetic changes, leading to long-term effects which last beyond drug treatment. Given that acquired resistance to BRAF inhibitors in melanoma is likely due to upregulation of alternative pathways, it is plausible that epigenetic regulation plays a role in acquired resistance [5]. In our experience, after stopping continuous dosing of vemurafenib, there is often rapid progression of disease. Here, the fact that this patient was treated with intermittent therapy could be an important factor in the ongoing complete response after ceasing therapy, since it might have changed the epigenetic profile of the melanoma in a beneficial way that could not be achieved with continuous dosing. Work in animal studies has shown that intermittent therapy can prevent acquired resistance [12]. Further research investigating whether response to treatment continues after ceasing intermittent vemurafenib and how intermittent treatment affects the epigenetic profile of tumours would be helpful.

\subsection{Immune Induction}

It is now clear that the immune system plays an important role in melanoma. Partial regression of primary cutaneous melanomas is a common event, though complete regression is rare [28]. Spontaneous regression of metastatic melanoma is very rare [29]. There are many potential mechanisms by which this might occur, but the most likely is the activation of the immune system to produce a greater than normal response. Many immunomodulating drugs have now been shown as effective treatments in melanoma. Interferon- $\alpha 2$ and interleukin 2 are approved for use in an adjuvant setting in selected cases [30, 31]. Ipilimumab is an anti-CTLA-4 monoclonal antibody which augments T-cell activation. Ipilimumab was the first drug to improve overall survival in the metastatic setting [32]. IMCgp100 is a novel immunotherapy currently in phase 1 trials [33]. Perhaps most significant has been the development of PD-1 inhibitors. Nivolumab and pembrolizumab are both PD-1 inhibitors approved for the treatment of melanoma in the US and in Europe [34-36].

Recently, there has been interest in whether inhibiting the MAPK pathway up-regulates the immune response against melanoma metastases. If this is the case, it could provide a possible explanation for the ongoing complete response after vemurafenib therapy was ceased in our patient. One study, in which biopsies were taken from patients before and after starting vemurafenib therapy, showed that BRAF inhibition was associated with increased melanoma antigen expression, increased CD8+ Tcell infiltrate and decreased immunosuppressive cytokines in tumours of patients with metastatic melanoma. When patients progressed, biopsies were again taken, showing that melanoma antigen expression and CD8+ T-cell infiltrate were decreased at the time of progression [37]. These findings would be consistent with a model where decreased immune response allows the tumour to escape immune surveillance and therefore progress. Animal studies have shown that host immunity can contribute to the antitumour activity of BRAF inhibition, with CCR2 suggested as an important participant [38]. It would be of interest to determine whether intermittent vemurafenib is associated with increased markers of an immune response compared with continuous vemurafenib. It would also be of interest 
to assess whether concurrent treatment with immunosuppressants, such as steroids, affects response to therapy with vemurafenib. Studies investigating the combination of BRAF inhibitors and immunotherapy in the treatment of metastatic melanoma are ongoing [38, 39]. Combined BRAF and MEK inhibition has now been found to be more effective that BRAF inhibition alone [40]. The effect of this combination on the immune system warrants further investigation.

It is worth noting that in our case, the patient suffered numerous toxicities which are possibly immune-related, particularly arthralgia. These continued despite dose reductions, and for some time after vemurafenib cessation. This may suggest that immune induction played a greater role in this patient than is typical with vemurafenib. That the response to therapy was rapid does not necessarily exclude an underlying immune component. Recent clinical trials combining ipilimumab and nivolumab in advanced melanoma have shown that over $80 \%$ reduction in target lesion size within 6-12 weeks is not uncommon [41].

\section{Summary}

In summary, over 24 months after ceasing intermittent vemurafenib therapy due to unacceptable toxicities, the patient described in this case remains well, with continued clinical complete response. To the best of our knowledge, a continued response of metastatic melanoma to vemurafenib after ceasing treatment has never before been described. Understanding how this occurred could provide greater insight into how vemurafenib exerts its effect in BRAF-mutant metastatic melanoma. We recommend follow-up of other patients who have had to cease vemurafenib due to unacceptable toxicities, in order to see how common the occurrence of continued response is after cessation of vemurafenib therapy, and whether the toxicity experienced can provide clues as to other mechanisms of action of this drug, beyond simple inhibition of the MAPK pathway.

\section{Compliance with Ethical Standards}

Funding MRM is supported by the Oxford Experimental Cancer Medicine Centre.

Conflict of Interest AG has received financial support from Bristol Myers Squibb for meeting attendance and payment for teaching educational webinars. MRM has received grants from Cancer Research UK, and also grants and/or personal fees from Amgen, Roche, AstraZeneca, GSK, BMS and Eisai, and study fees (institution only) from Novartis, Astellas, Millennium, Immunocore, BMS, Vertex, Eisai, AbbVie, Clovis, Pfizer and Merck.

Consent Written informed consent was obtained from the patient for her data to be used anonymously for teaching and research purposes.

\section{References}

1. Davies H, Bignell GR, Cox C, Stephens P, Edkins S, Clegg S, et al. Mutations of the BRAF gene in human cancer. Nature. 2002;417: 949-54.

2. Fecher LA, Amaravadi RK, Flaherty KT. The MAPK pathway in melanoma. Curr Opin Oncol. 2008;20:183-9.

3. Chapman PB, Hauschild A, Robert C, Haanen JB, Ascierto P, Larkin J, et al. Brim-3 study group. improved survival with vemurafenib in melanoma with BRAF V600E mutation. N Engl J Med. 2011:364:2507-16.

4. Hauschild A, Grob J-J, Demidov LV, Jouary T, Gutzmer R, Millward M, et al. Dabrafenib in BRAF-mutated metastatic melanoma: a multicentre, open-label, phase 3 randomised controlled trial. Lancet. 2012;380:358-65.

5. Sullivan RJ, Flaherty KT. Resistance to BRAF-targeted therapy in melanoma. Eur J Cancer. 2013;49:1297-304.

6. McArthur GA, Chapman PB, Robert C, Larkin J, Haanen JB, Dummer R, et al. Safety and efficacy of vemurafenib in BRAF(V600E) and BRAF(V600K) mutation-positive melanoma (BRIM-3): extended follow-up of a phase 3, randomised, openlabel study. Lancet Oncol. 2014;15:323-32.

7. Scholtens A, Geukes Foppen MH, Blank CU, van Thienen JV, van Tinteren H, Haanen JB. Vemurafenib for BRAF V600 mutated advanced melanoma: results of treatment beyond progression. Eur J Cancer. 2015;51:642-52.

8. Soria JC, Wu YL, Nakagawa K, Kim SW, Yang JJ, Ahn MJ, et al. Gefitinib plus chemotherapy versus placebo plus chemotherapy in EGFR-mutation-positive non-small-cell lung cancer after progression on first-line gefitinib (IMPRESS): a phase 3 randomised trial. Lancet Oncol. 2015;16:990-8.

9. Jackman D, Pao W, Riely G, Kris M, Janne P, Lynch T, et al. Clinical definition of acquired resistance to epidermal growth factor receptor tyrosine kinase inhibitors in non-small-cell lung cancer. J Clin Oncol. 2010;28:357-60.

10. Gainor JF, Shaw AT. Emerging paradigms in the development of resistance to tyrosine kinase inhibitors in lung cancer. J Clin Oncol. 2013:31:3987-96.

11. Dooley AJ, Gupta A, Bhattacharyya M, Middleton MR. Intermittent dosing with vemurafenib in BRAF V600E-mutant melanoma: review of a case series. Ther Adv Med Oncol. 2014;6:262-6.

12. Das Thakur M, Salangsang F, Landman AS, Sellers WR, Pryer NK, Levesque MP, et al. Modelling vemurafenib resistance in melanoma reveals a strategy to forestall drug resistance. Nature. 2013;494: 251-5.

13. Faries MB, Steen S, Ye X, Sim M, Morton DL. Late recurrence in melanoma: clinical implications of lost dormancy. J Am Coll Surg. 2013;217:27-34.

14. Dietrich S, Huellein J, Hundemer M, Lehners N, Jethwa A, Capper $\mathrm{D}$, et al. Continued response off treatment after BRAF inhibition in refractory hairy cell leukemia. J Clin Oncol. 2013;31:E300-3.

15. Tiacci E, Trifonov V, Schiavoni G, Holmes A, Kern W, Martelli MP, et al. BRAF mutations in hairy-cell leukemia. N Engl J Med. 2011;364:2305-15.

16. Sullivan RJ, Flaherty K. MAP kinase signaling and inhibition in melanoma. Oncogene. 2013;32:2373-9.

17. Tiacci E, Liso A, Piris M, Falini B. Evolving concepts in the pathogenesis of hairy-cell leukaemia. Nat Rev Cancer. 2006;6:437-48.

18. Mozūraitienė J, Bielskienė K, Atkočius V, Labeikytė D. Molecular alterations in signal pathways of melanoma and new personalized treatment strategies: targeting of Notch. Medicina. 2015;51:13345 .

19. Bresnick AR, Weber DJ, Zimmer DB. S100 proteins in cancer. Nat Rev Cancer. 2015;15:96-109. 
20. Chapman PB. Am Soc Clin Oncol Educ Book. 2013. doi:10.1200/ EdBook_AM.2013.33.e80

21. Tiacci E, Trifonov V, Schiavoni G, Holmes A, Kern W, Martelli. BRAF mutations in hairy cell leukemia. N Engl J Med. 2011;364: 2305-15.

22. Langabeer SE. Exceptions to the rule in hairy cell leukaemia: implications for molecular diagnostics and targeted therapy. Med Oncol. 2014;31:895.

23. Polak P, Karlić R, Koren A, Thurman R, Sandstrom R, Lawrence MS, et al. Cell-of-origin chromatin organization shapes the mutational landscape of cancer. Nature. 2015;518:360-4.

24. Yancovitz M, Litterman A, Yoon J, Ng E, Shapiro RL, Berman RS, et al. Intra- and inter-tumor heterogeneity of BRAF(V600E) mutations in primary and metastatic melanoma. Plos One. 2012;7(1), e29336. doi:10.1371/journal.pone.0029336

25. Mervic L. Time course and pattern of metastasis of cutaneous melanoma differ between men and women. Plos One. 2012;7(3), e32955. doi:10.1371/journal.pone.0032955

26. Valdespino V, Valdespino PM. Potential of epigenetic therapies in the management of solid tumors. Cancer Manag Res. 2015;31:241-51.

27. Renthal W, Nestler EJ. Epigenetic mechanisms in drug addiction. Trends Mol Med. 2008;14:341-50.

28. Blessing K, McLaren KM. Histological regression in primary cutaneous melanoma: recognition, prevalence and significance. Histopathology. 1992;20:315-22.

29. Kalialis LV, Drzewiecki KT, Klyver H. Spontaneous regression of metastases from melanoma: review of the literature. Melanoma Res. 2009;19:275-82.

30. Mocellin S, Pasquali S, Rossi CR, Nitti D. Interferon alpha adjuvant therapy in patients with high-risk melanoma: a systematic review and meta-analysis. J Natl Cancer Inst. 2010;102:493-501.

31. Petrella T, Quirt I, Verma S, Haynes AE, Charette M, Bak K, et al. Single-agent interleukin-2 in the treatment of metastatic melanoma: a systematic review. Cancer Treat Rev. 2007;33:484-96.
32. Hodi FS, O’Day SJ, McDermott DF, Weber RW, Sosman JA, Haanen JB, et al. Improved survival with Ipilimumab in patients with metastatic melanoma. N Engl J Med. 2010;363:711-23.

33. Bossi G, Buisson S, Oates J, Jakobsen BK, Hassan NJ. ImmTACredirected tumour cell killing induces and potentiates antigen crosspresentation by dendritic cells. Cancer Immunol Immunother. 2014;63:437-48.

34. Hamid O, Robert C, Daud A, Hodi FS, Hwu W-J, Kefford R, et al. Safety and tumor responses with Lambrolizumab (Anti-PD-1) in melanoma. N Engl J Med. 2013;369:134-44.

35. US Food and Drug Administration. (2014). Pembrolizumab. Available at: http://www.fda.gov/Drugs/InformationOnDrugs/ ApprovedDrugs/ucm412861.htm. (accessed 31 August 2015).

36. News in Brief: PD-1 inhibitor approved for melanoma. Cancer Discov. 2014;4:1249.

37. Frederick DT, Piris A, Cogdill AP, Cooper ZA, Lezcano C, Ferrone $\mathrm{CR}$, et al. BRAF inhibition is associated with enhanced melanoma antigen expression and a more favorable tumor microenvironment in patients with metastatic melanoma. Clin Cancer Res. 2013;19: 1225-31.

38. Knight DA, Ngiow SF, Li M, Parmenter T, Mok S, Cass A, et al. Host immunity contributes to the anti-melanoma activity of BRAF inhibitors. J Clin Invest. 2013;123:1371-81.

39. Hu-Lieskovan S, Robert L, Moreno BH, Ribas A. Combining targeted therapy with immunotherapy in BRAF-mutant melanoma: promise and challenges. J Clin Oncol. 2014;32:224854.

40. Robert C, Karaszewska B, Schachter J, Rutkowski P, Mackiewicz A, Stroiakovski D, et al. Improved overall survival in melanoma with Combined Dabrafenib and Trametinib. N Engl J Med. 2015;372:30-9.

41. Wolchok JD, Kluger H, Callahan MK, Postow MA, Rizvi NA, Lesokhin AM, et al. Nivolumab plus ipilimumab in advanced melanoma. N Engl J Med. 2013;369:122-33. 